\title{
Rigorous engineering of product-line requirements: a case study in failure management
}

\author{
Colin Snook $^{\mathrm{a}}$, Michael Poppleton ${ }^{\mathrm{a}, *}$, Ian Johnson ${ }^{\mathrm{b}}$ \\ ${ }^{a}$ School of Electronics and Computer Science, University of Southampton, Highfield, \\ Southampton SO17 1BJ, UK \\ ${ }^{\mathrm{b}}$ AT Engine Controls, Portsmouth
}

\begin{abstract}
We consider the failure detection and management function for engine control systems as an application domain where product line engineering is indicated. The need to develop a generic requirement set - for subsequent system instantiation - is complicated by the addition of the high levels of verification demanded by this safety-critical domain, subject to avionics industry standards. We present our case study experience in this area as a candidate method for the engineering, validation and verification of generic requirements using domain engineering and Formal Methods techniques and tools. For a defined class of systems, the case study produces a generic requirement set in UML and an example system instance. Domain analysis and engineering produce a validated model which is integrated with the formal specification/ verification method B by the use of our UML-B profile. The formal verification both of the generic requirement set, and of a simple system instance, is demonstrated using our U2B, ProB and prototype Requirements Manager tools.
\end{abstract}

This work is a demonstrator for a tool-supported method which will be an output of EU project RODIN ${ }^{1}$. The use of existing and prototype formal verification and support tools is discussed. The method, developed in application to this novel combination of product line, failure management and safety-critical engineering, is evaluated and considered to be applicable to a wide range of domains.

Key words: formal specification, generic requirements, product line, refinement, tools, UML-B, verification

\footnotetext{
1 This work is conducted in the setting of the EU funded research project: IST 511599 RODIN (Rigorous Open Development Environment for Complex Systems) http://rodin.cs.ncl.ac.uk/.

* Corresponding author

Email addresses: cfseecs. soton.ac.uk (Colin Snook), mrp@ecs.soton.ac.uk (Michael Poppleton), IJohnson@atenginecontrols.com (Ian Johnson).
} 


\section{Introduction}

The notion of software product line (also known as system family) engineering became well established [29], after Parnas' proposal [35] in the 70's of information hiding and modularization as techniques that would support the handling of program families. Product line engineering arises where multiple variants of essentially the same software system are required, to meet a variety of platform, functional, or other requirements. This kind of generic systems engineering is well known in the avionics industry; e.g. [25,19] describe the reuse of generic sets of requirements in engine control and flight control systems.

Domain analysis and object oriented frameworks are among numerous solutions proposed to product line technology. In Domain-Specific Software Architecture [44] for example, the domain engineering of a set of general, domain-specific requirements for the product line is followed by its successive refinement, in a series of system engineering cycles, into specific product requirements. On the other hand [20] describes the Object-Oriented Framework as a "a reusable, semi-complete application that can be specialized to produce custom applications". Here the domain engineering produces an object-oriented model that must be instantiated, in some systematic way, for each specific product required. In this work we combine objectoriented and formal techniques and tools in domain and product line engineering.

Developers in the avionics industry are interested in the use of object-oriented and UML technology (OOT) [11,32] as a way to increase productivity. Concepts such as inheritance and design patterns facilitate the reuse of requirements and designs. UML has emerged as the de-facto standard modelling language for object-oriented design and analysis, supported by a wide variety of tools. Due to concerns over safety certification issues however, OOT has not seen widespread use in avionics applications. The controlling standards used in the industry, such as RTCA DO178B [21] and its European equivalent, EUROCAE ED-12B [1], do not consider OOT, although this is under review.

It is widely recognized that formal methods (FM) technology makes a strong contribution to the verification required for safety-critical systems; indeed, DefStan 00-55 [33] as well as the avionics standards above recommend the use of FM for critical systems. It is further recognized that FM will need to be integrated [5] in as "black-box" as possible a manner - with OOT in order to achieve serious industry penetration. The combination of UML and formal methods therefore offers the promise of improved safety and flexibility in the design of software for aviation certification.

One approach to the integration of FM and OOT is to enhance - at the abstract modelling stage - UML with the minimum amount of textual formal specification required to completely express functional, safety and other requirements. A tool 
will convert the customer-oriented abstract UML model to a fully textual model as input to FM tools such as model-checkers and theorem provers. With suitable tool support for configuration and project management, this approach will facilitate the reuse of verified software specifications and consequently code components.

Adoption of formal methods in the safety-critical industry has been limited partly due to the need for industrial strength tool support. The B method of J.-R. Abrial [2,39] is a formal method with good tool support, and a good industrial track record, e.g. [17]. An approach that integrates formal specification and verification in the B language, with UML-based design methods, has been under development at Southampton for some years. The UML-B [42] is a profile of UML that defines a formal modelling notation combining UML and B. It is supported by the U2B tool [40], which translates UML-B models into B, for subsequent formal verification. This verification includes model-checking with the ProB model-checker [27] for B. These tools have all been developed at Southampton, and continue to be extended in current work in project RODIN, which aims to produce the next-generation EventB method and tools.

\subsection{Failure detection and management (FDM) for engine control}

A common functionality required of many systems is tolerance to faults in the environment, i.e. the detection and management of failed of input signals. This is particularly pertinent in aviation applications where lack of tolerance to failed system inputs could have severe consequences. The failure manager filters environmental inputs to the control system, providing the best information possible whilst determining whether a component has failed or not. The role of failure management in an embedded control system is shown in Fig. 1.

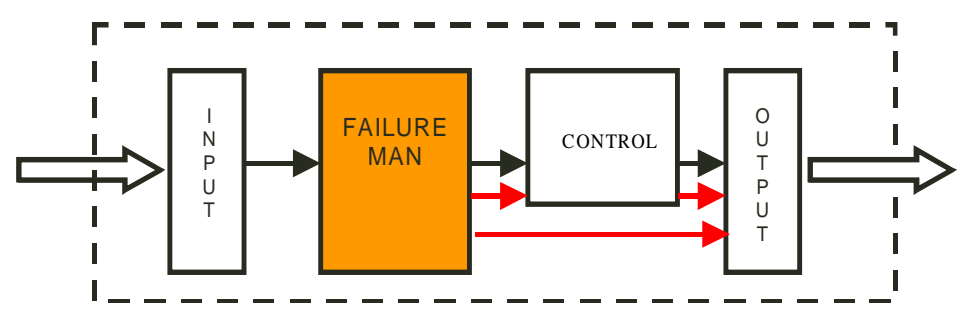

Fig. 1. Context diagram for failure management subsystem

Inputs may be tested for signal magnitude and/or rate of change being within defined bounds, and for consistency with other inputs. If no failure is detected, some input values are passed on to the control subsystem; others are only used for failure detection and management. When a failure is detected it is managed in order to maintain a usable set of input values for the control subsystem. This may involve substituting values, and taking alternative actions. To prevent over-reaction to isolated transient values, a failed condition must persist for a period of time before a failure is confirmed. If the invalid condition is not confirmed the input recovers and 
is used again. Temporary remedial actions, such as relying on the last good value, or suppressing control behaviour, may be taken while a failure is being confirmed. Once a failure is confirmed, more permanent actions are taken such as switching to an alternative source, altering or degrading the method of control, engaging a backup system or freezing the controlled equipment.

\subsection{Contribution}

Failure detection and management (FDM) in engine control systems is a demanding application domain, see e.g. [10]. Based on work on a case study provided by AT Engine Controls, we propose a method for the engineering, validation and verification of generic requirements for product-line purposes. The approach exploits genericity both within as well as between target system variants. Although productline engineering has been applied in engine and flight control systems [25,19], we are not aware of any such work in the FDM domain. Using UML-B we define generic classes of failure-detection test for sensors and variables in the system environment, such as rate-of-change, limit, and multiple-redundant-sensor, which are simply instantiated by parameter. Multiple instances of these classes occur in any given system. Failure confirmation is then a generic abstraction over these test classes: it constitutes a configurable process of execution of specified tests over a number of system cycles, that will determine whether a failure of the component under test has occurred.

A complicating factor is the instability of the FDM requirements domain, which is often subject to late change. This is because the failure characteristics of dynamic controlled systems are usually dependent on interaction with the control systems being developed and can only be fully determined via prototyping. Our generic requirements formulation accommodates this ongoing requirements change process.

Our approach contributes methodologically to product-line requirements engineering in its integration of informal domain analysis with domain and application engineering that exploits both UML and Formal Methods technology. The application of product-line engineering to failure detection and management is also novel. We have exercised the first three stages of our proposed four-stage process model: (i) domain analysis developed a generic requirement specification document for a class of systems, (ii) domain engineering then validated the requirements, producing a formal generic model in UML-B for the product line, and (iii) application engineering then verified an example instance system. The fourth stage of the method, the addition and verification of behaviour to generic and instance models, is ongoing work. 


\subsection{Structure of the paper}

The paper proceeds as follows. Section 2 introduces formal specification and verification in B, and our approach in Southampton. Section 3 gives an overview of our method. Sections 4 - 5 discuss the domain analysis and engineering activities that result in a validated generic model. Section 6 discusses the application engineering of an instantiated system variant to verify the instance model. Section 7 gives an industrial user's perspective on the usability and applicability of the method and tools. Finally section 8 concludes with a discussion of related and future work, and an evaluation of the method.

\section{Formal specification and verification with $B$}

The B language [2] of J.-R. Abrial is a wide-spectrum language supporting a full formal development lifecycle from specification through refinement to programming. It is supported by full-lifecycle verification toolkits such as Atelier B [4], and has been instrumental in successful safety-critical system developments such as signalling on the Paris Metro [17].

A B specification gives an abstract description of requirements, modelled in terms of system state and behaviour. Simply put, state is described in terms of sets and relations on those sets, and behaviour in terms of changes to that state caused by invocation of events or operations. An invariant clause captures required properties of the system that must hold at all times, defining the meaning of the data and the integrity of its structure. The B verification tools [12] generate proof obligations (POs) that initialization and all operations maintain the invariant; this is called operation consistency. Automatic and interactive provers are part of the method; we do not discuss them further here.

A refinement step involves the transformation of an early, abstract nondeterministic specification into a more concrete one ${ }^{2}$, by elaboration with more data and algorithmic structure, thus reducing nondeterminism. Using the refinement relation between abstract and concrete models, proof obligations guarantee that the refinement correctly represents the behaviour of the abstract specification it refines.

\subsection{The Southampton approach}

At Southampton two tools have been developed to support formal system development in B: ProB and U2B. ProB [27] is a model checker that searches the full

$\overline{2}$ The concrete specification in this context is often called the refinement. 
abstract state machine model of a B specification for invariant violations, returning any counterexample found. The state model can be presented graphically. Model checking avoids the effort of proof debugging in early development activities, serving as a preliminary validation of the specification before commencing proof. ProB furthermore provides a limited form of temporal model-checking, and user-driven animation of behaviour. Its use is discussed further in sections 5 and 6 .

The UML-B [42] is a profile of UML that defines a formal modelling notation. It has a mapping to, and is therefore suitable for translation into, the B language. UML-B consists of class diagrams with attached statecharts, and an integrated constraint and action language called $\mu \mathrm{B}$, based on $\mathrm{B}$. UML-B is thus comparable to the UML/OCL specification approach of USE [22,23]. The profile uses stereotypes to specialise the meaning of UML entities to enhance correspondence with B concepts. UML-B provides a diagrammatic, formal modelling notation based on UML. The popularity of the UML enables UML-B to overcome some of the barriers to the acceptance of formal methods in industry. Its familiar diagrammatic notations make specifications accessible to domain experts who may not be familiar with formal notations. UML-B hides B's infrastructure by packaging mathematical constraints and action specifications in $\mu \mathrm{B}$ into small sections within the context of an owning UML entity. The U2B [40] translator converts UML-B models into B components (abstract machines and their refinements), thus enabling B verification and validation technology to be exploited.

\section{Overview of method}

We first give an overview of the method which is then discussed in more detail in the following sections - see Fig. 2. The first stage is domain analysis (section 4) which is based on prior experience of developing products for the application domain of failure detection and management in engine control. This domain analysis is guided by the experience of [25], who also worked in the engine control domain. Its purpose is twofold: (i) to "identify reusable artifacts in the domain", and (ii) to define a taxonomy of generic requirements and produce a generic requirements specification document (RSD) [6] subject to that taxonomy ${ }^{3}$. A first-cut generic model in object-association terms, naming and relating these generic requirements, is constructed as part of the RSD.

The identification of a useful generic model is a difficult process and therefore further validation and development of the model is required. This is done in the domain engineering stage (section 5) where a more rigorous examination of the first-cut model is undertaken, using the B-method and the Southampton tools. This stage also serves to structure "the reusable artifacts in such a way (sic) that facilitated

$\overline{3}$ Experience [3] shows the value of this taxonomic approach to requirements specification. 
reuse during the development of new applications" [25]. The model is animated by creating typical instances of its generic requirement entities, to test when it is and is not consistent. This stage is model validation by animation, using the ProB and U2B tools, to show that it is capable of holding the kind of information that is found in the application domain. During this stage the relationships between the entities are likely to be adjusted as a better understanding of the domain is developed. This stage results in a validated generic model of requirements that can be instantiated for each new application.

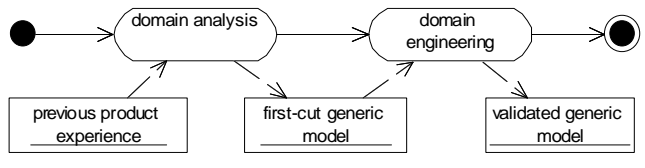

Fig. 2. Process for obtaining the generic model

For each new application instance, the requirements are expressed as instances of the relevant generic requirement objects and their associations, in an instance model - see Fig. 3. The ProB model checker is then used to verify that the application is consistent with the relationship constraints embodied in the generic model. This instance, or application engineering stage, producing a verified consistent instance model, shows that the requirements are a consistent set of requirements for the domain. It does not, however, show that they are the right (desired) set of requirements, in terms of system behaviour that will result.

The final stage, therefore, is to add dynamic features to the instantiated model in the form of variables and operations that model the behaviour of the entities in the domain and to animate this behaviour so that the instantiated requirements can be validated. This final stage of the process - "validate instantiation" in Fig. 3 - is work in progress.

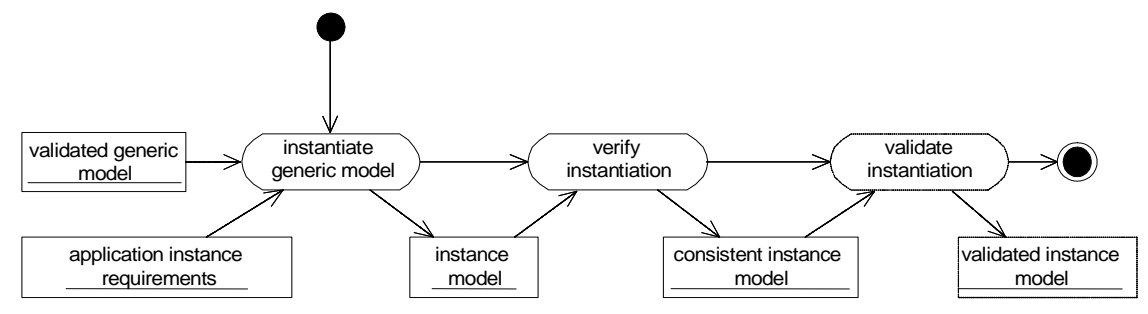

Fig. 3. Process for using the generic model in a specific application

Each method stage will be followed by an instance of a stage template. This will summarize and structure the stage by briefly identifying its approach, parameters and $\mathrm{V} \& \mathrm{~V}$ activities, as well as stage inputs and outputs:

Inputs: Documents, information, people consulted as input to this stage Approach: Techniques or procedures applied Parameters: Any data specific to this stage 
Outputs: Documents and results produced by this stage

$\mathbf{V} \& \mathbf{V}$ : Validation/ verification activities applied during this stage

\section{Domain analysis}

The strategy adopted to reach the first-cut generic requirement model (Fig. 2) was to apply domain analysis in a style similar to that used by Lam [25]. Prieto-Diaz [37] defines domain analysis as "a process by which information used in developing software systems is identified, captured and organised with the purpose of making it reusable when creating new systems". He identifies three activities which are central to our domain analysis:

(1) identification of reusable entities

(2) abstraction or generalization

(3) classification and cataloging for further reuse

The first step was to define the scope of the domain in discussion with the engine controller experts. This was done by considering legacy specification documents of a small number of representative failure management engine systems, resulting in a brief scoping definition of the FMS. This definition forms an early part of the requirements specification document (RSD) produced by this project and is given in table 1.

Table 1

FMS scoping requirements

\begin{tabular}{|l|l|}
\hline PROC1 & The subsystem executes on a given process cycle. \\
\hline DET1 & The subsystem detects abnormal conditions of inputs caused by failures of the external equipment. \\
\hline OUT1 & $\begin{array}{l}\text { Inputs that are found to be in a normal condition may be passed on as outputs (if they are required } \\
\text { by other subsystems). }\end{array}$ \\
\hline CONF1 & $\begin{array}{l}\text { When an abnormal condition is detected, the subsystem confirms the suspected failure over a } \\
\text { period of time. During this time the condition may recover. }\end{array}$ \\
\hline ACT1 & $\begin{array}{l}\text { The subsystem takes some temporary action to simulate acceptable input while a suspected fault } \\
\text { is being confirmed. }\end{array}$ \\
\hline PROC2 & $\begin{array}{l}\text { The subsystem simulates acceptable input conditions or performs other permanent failure actions } \\
\text { if it confirms an abnormal condition of the inputs. } \\
\text { to meet the specific requirements of the application (as shown in ...). }\end{array}$ \\
\hline
\end{tabular}

Identification of commonalities (reusable entities) by abstraction: Legacy documents were used, with expert consultation, to identify specific requirements that might reveal more abstractly defined requirements generic across airframes. That is, specific examples were sought of logical groups of airframe instance requirements, where a group aggregated similar requirements from various system instances. Each group was then used to identify a higher-level commonality, by a process of abstraction. Table 2 shows three of these specific requirement/ commonality pairs, giving just one of the instance requirements that resulted in the 
associated commonality.

Table 2

Sample instance requirements revealing commonalities

\begin{tabular}{|l|l|}
\hline (1) Engine speed ES & $\begin{array}{l}\text { Each ES sensor ESa, ESb has one upper bound and two lower bounds for range check- } \\
\text { ing. The upper bound test is valid at all times. Lower bound test 1 (starting) is valid } \\
\text { if input condition START_MODE and LIGHT_OFF and not START_ABORT. Lower } \\
\text { bound test 2 (running) is valid if input condition RUN_MODE and not START_MODE. }\end{array}$ \\
\hline & $\begin{array}{l}\text { An INPut is subject to a number of tests; each such test is subject to a CONDition (over } \\
\text { INPuts, in this case). }\end{array}$ \\
\hline (2) Engine torque EQ & $\begin{array}{l}\text { EQ has a lower bound test valid if output condition ES }>80 \% \text {. This test is subject to a } \\
\text { long fault count } 2-1-32 \text {. That is, each fault adds +2, each non-fault adds -1 to a fault } \\
\text { count subject to } 0=\text { pass, 32 = fail. }\end{array}$ \\
\hline $\begin{array}{l}\text { A test is subject to a CONDition over an OUTput; each such test is subject to a DETec- } \\
\text { tion and a CONFirmation fault count. }\end{array}$ \\
\hline (3) Engine speed ES & $\begin{array}{l}\text { Output engine speed cES is set to ESa only when all 3 magnitude DETections, 1 differ- } \\
\text { ence DETection, and one rate DETection are passed. }\end{array}$ \\
\hline & An OUTput is set by an ACTion, where that ACTion results from a successful test. \\
\hline
\end{tabular}

This analysis quickly revealed a high degree of genericity in the static configuration of the system: a small number of key system entities were quickly revealed in a fixed relation to each other. In table 2 (1), the engine speed sensor is an INPut entity subject to a number of tests or DETections. A DETection is a predicate that compares the value of an expression involving the input to a limit value, e.g. a range check. Each test is invoked subject to a defined CONDition - a predicate based on the values and/or failure states of other INPut sensors. In (2) we see that a test consitutes both a DETection and a CONFirmation, that is the application of an iterative algorithm to confirm, over a number of sampling cycles, whether an input's failed status is transient or permanent. The configuration of a specific system instance will be defined by an instantiation of these entities with instance sensor fits, detection parameters etc.

Generic requirements specification: In the emerging RODIN method for EventB [31] Abrial recommends labelling requirements in the RSD taxonomically; in our case the taxonomy emerged naturally from the generic entities revealed by the abstraction process above:

INP Input to be tested.

COND Condition under which a test is performed. A predicate over INP and OUT values.

DET Detection of a failure state. A predicate that compares the value of an expression involving the input to a limit value.

CONF Confirmation of a (persistent) failure state.

ACT Action taken either normally or in response to a failures, possibly subject to a condition. Assigns the value of an expression, which may involve inputs and/or other output values, to an output.

OUT Output signal to be used in an action 
Elaboration of relationships: The analysis then elaborated the entity relationships. This was used to form the first-cut generic model of Fig. 2, which is elaborated in UML in Fig. 4. An input (INP) instance represents a sensor value input from the environment. It may have many associated tests, and a test may utilise many other inputs. A TEST is composed of a detection method (DET) and confirmation mechanism (CONF) pair ${ }^{4}$. For example an engine speed input, which is tested for out of range magnitude as well as excessive rate of change, has a detection and associated confirmation for the magnitude test and a different detection and different confirmation for the rate of change test. Each test also has a collection of conditions (COND) that must be satisfied for the test to be applied. For example, the engine speed magnitude test that is applied when the engine is running is different from the engine speed magnitude test applied while starting the engine. A confirmation mechanism is associated with three different sets of actions (ACT), the healthy actions (hAct), the temporary actions ( $t A c t$ ), taken while a test is confirming failure, and the permanent actions ( $\mathrm{pAct}$ ), taken when a test has confirmed failure. Each action is associated with at least one output (OUT) that it modifies.

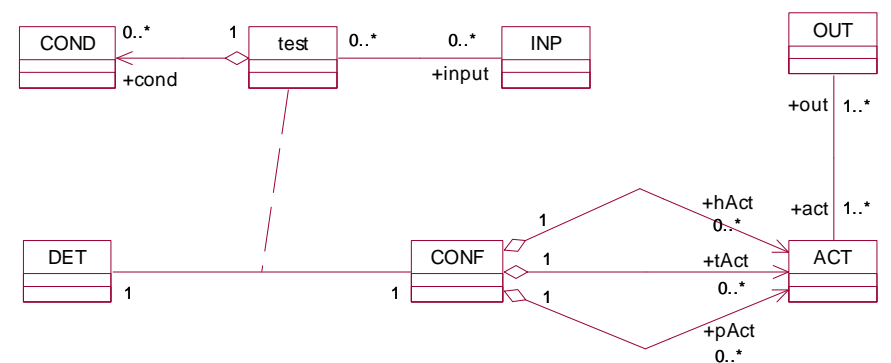

Fig. 4. Overview of common types of elements of functionality and their interrelationships

The detection mechanism DET of a test can be further decomposed as (i) a check (DET_MAG) that signal magnitude is within defined bounds, (ii) a check (DET_RATE) that signal rate of change is within defined bounds or (iii) a comparison (DET_PRED) with a predicted value.

Requirements specification document (RSD): The generic requirements were recorded into a traceable requirements specification document [6] for the case study. The document had several features which assisted in presenting the requirements in a way suitable for further analysis, in particular a generic section and an example instantiation of it in tabular form. Note that every system instance will require specification in such a tabular form.

The generic section of the RSD includes

(1) The taxonomy of requirements.

(2) The model (Fig.4) of the generic requirement domain.

$\overline{4}$ Consequently TEST is not a category in the requirements taxonomy above. 
(3) For each labelled generic requirement, a concise statement of the requirement and an explanation of the rationale behind it.

Table 3 gives some examples of the resulting generic requirements from the RSD.

Table 3

Sample generic requirements

\begin{tabular}{|c|c|}
\hline INP2 & The subsystem uses input variables which contain either digitized values or Boolean states. \\
\hline DET_MAG1 & $\begin{array}{l}\text { Compares input value against a magnitude (range) limit. The input is in error if the limit is ex- } \\
\text { ceeded. }\end{array}$ \\
\hline DET_PRED1 & $\begin{array}{l}\text { Compare input value against a computed value. The input is in error if the discrepancy lies outside } \\
\text { a tolerance of this value. }\end{array}$ \\
\hline DET_CONF1 & $\begin{array}{l}\text { Every detection is subject to precisely one confirmation; every confirmation acts on precisely one } \\
\text { detection. }\end{array}$ \\
\hline DET_COND1 & Every detection may act subject to a number of conditions \\
\hline CONF4 & $\begin{array}{l}\text { (persistence counter failure mechanism) If a test has detected an error (failure) on an input then } \\
\text {...(algorithm) }\end{array}$ \\
\hline
\end{tabular}

The example instantiation section is in tabular form and consists of uniquely identified instances of the elements in the generic model, and references from each instance to other instances as described by the relationships in the generic model. Table 4 gives two instances from each of DET_MAG and CONF data tables:

Table 4

Sample instance requirements

\begin{tabular}{|l|l|l|l|l|l|l|l|}
\hline Ref. & $\begin{array}{l}\text { value } \\
\text { tested }\end{array}$ & name & dir & limit & freq & condition & $\begin{array}{l}\text { confirm } \\
\text { ms }\end{array}$ \\
\hline MAG1.21 & INP2.10 & ESa & lo & 45 & 24 & COND5.3 & CONF4.1 \\
\hline MAG1.22 & INP2.11 & Esb & up & 130 & 24 & COND1 & CONF4.1 \\
\hline \hline Ref. & name & x inc & $\mathbf{y}$ dec & z limit & description \\
\hline CONF4.1 & fault count 2-1-8 & 2 & 1 & 8 & $\begin{array}{l}\text { fault counter with } \\
\text { bias to confirm }\end{array}$ \\
\hline CONF4.5 & fault count 2-1-32 & 2 & 1 & 32 & $\begin{array}{l}\text { biased fault counter, } \\
16 \text { to confirm }\end{array}$ \\
\hline
\end{tabular}

Key issues and rationale for requirements: Concurrently with the above activity, we established key issues, or high-level requirements goals. Key issues were identified which served as "higher-level" properties required of the system. An example of such a key property would be that the failure management system must not be held in a transient action state indefinitely. Considering the requirements' rationale is useful in reasoning about requirements in the domain [25]. The rationale from which the above property has been derived, is that a transient state is temporary and actions associated with this state may only be valid for a limited time. Table 5 gives some further examples.

Reflection: This analysis showed that failure management systems are characterised by a high degree of fairly simple similar units made complex by a large 
Table 5

Key issues and rationale

\begin{tabular}{|l|l|}
\hline (1) Detect failures & $\begin{array}{l}\text { Outsource environment failure detection/ sanitization from } \\
\text { control system to FMS. Detect possible failures in input sig- } \\
\text { nals from airframe with maximum sensitivity, ie maximum } \\
\text { true positives. }\end{array}$ \\
\hline $\begin{array}{l}\text { (2) Confirm failure before perma- } \\
\text { nent action is taken }\end{array}$ & $\begin{array}{l}\text { Tolerate noise in the input signals: transient signal deviations } \\
\text { will be tolerated with maximum specificity, i.e. maximum true } \\
\text { negatives. }\end{array}$ \\
\hline (3) Take action appropriately & $\begin{array}{l}\text { Once a failure has been detected some action to cater for the } \\
\text { failure must be taken. The most appropriate action may depend } \\
\text { on the conditions of other inputs and outputs. }\end{array}$ \\
\hline $\begin{array}{l}\text { (4) Apply tests under appropriate } \\
\text { conditions }\end{array}$ & $\begin{array}{l}\text { Some tests are only valid under certain conditions of other in- } \\
\text { puts and outputs and could otherwise result in false positives. }\end{array}$ \\
\hline
\end{tabular}

number of minor variations and interdependencies. The domain presents opportunities for a high degree of reuse within a single product as well as between products. For example, a magnitude test type is usually required in a number of instances in a particular system. This is in contrast to the engine start domain addressed by Lam [25], where a single instance of each reusable function exists in a particular product. The method described in this paper is targeted at domains such as failure management where a few simple units are reused many times and a particular configuration depends on the relationships between the instances of these simple units. We will return to the applicability of the method in conclusion in section 8 .

\section{Domain analysis - stage template:}

Inputs: Engine controller experts. Legacy specification documents of representative failure management engine systems

Approach: Identification of commonalities by abstraction. Elaboration of relationships. Generic requirements specification. Identification of requirements' key issues and rationales

Parameters: UML class modelling. Abrial's taxonomic approach to requirements specification

Outputs: Generic requirements specification document (RSD) including first-cut UML model

V \& V: Informal peer review

\section{Domain Engineering}

The aim of the domain engineering stage is to validate the first-cut generic model of the requirements, thus deriving a validated generic requirements model as per Fig. 2. At input to the domain engineering stage this is essentially a pure UML class model (Fig. 4) without any dynamic features. The first task is conversion of this model to UML-B (Fig. 5), to enable automatic generation of the B model. The main work of this stage is the considered validation and derivation of the output model 
using ProB, resulting in our case in the model shown in Fig.6. Of course, since these models are diagrammatic representations of the narrative generic requirements, this stage also updates and corrects those requirements.

We will illustrate this derivation process by trying it with specific example scenarios taken from existing applications. In this way, we develop our understanding of the common, reusable elements within the domain by testing the relationships between them. Again, we rely on our knowledge of existing systems.

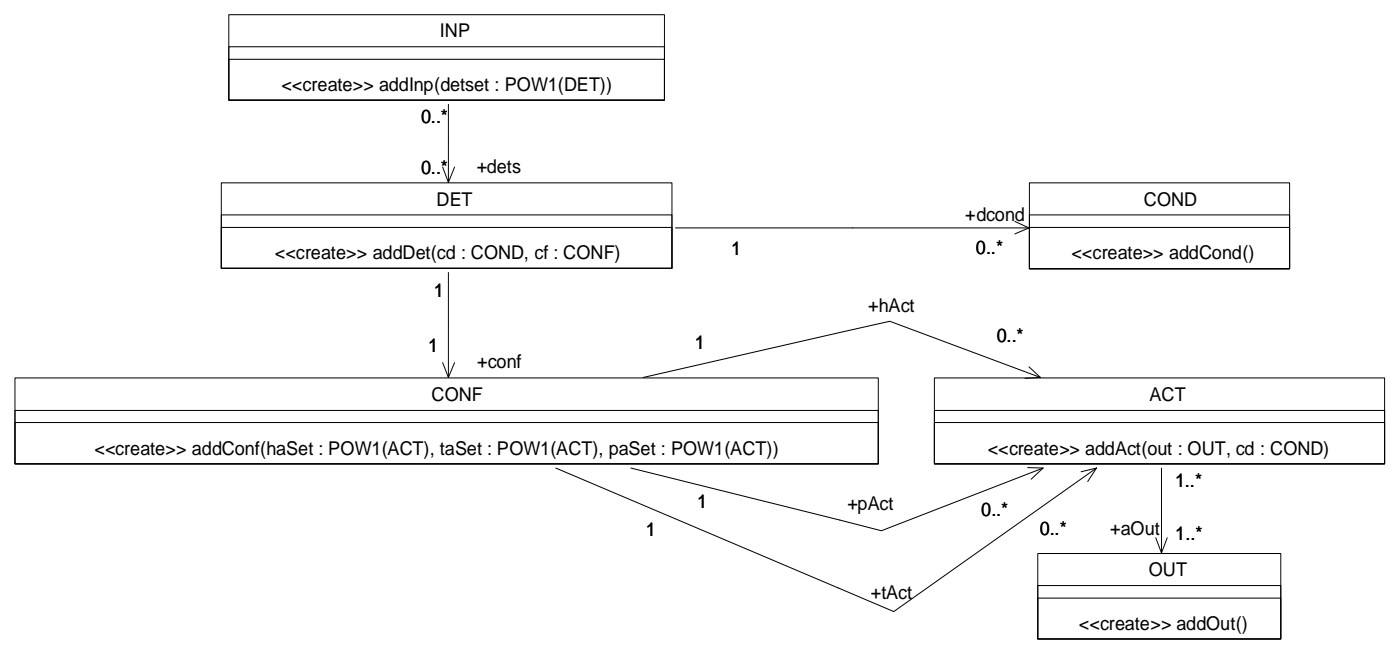

Fig. 5. Initial UML-B version of generic requirements model

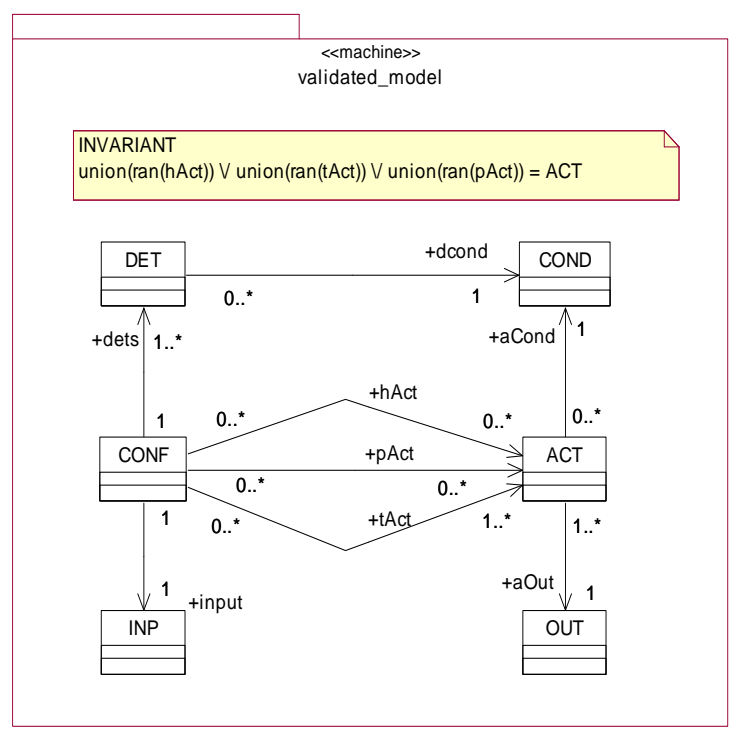

Fig. 6. Final UML-B version of generic requirements model

Conversion to UML-B: The first-cut model from the domain analysis was converted to the UML-B notation (Fig. 5) by making associations directional and 
adding stereotypes and UML-B clauses (tagged values) as defined in the UMLB profile [42]. U2B does not support association classes in a suitable form for the following validation. Therefore, before starting we removed the association class TEST. Instead we (i) mapped association dcond from class DET to class COND, (ii) removed association input, and (iii) mapped association dets from INP to DET.

Conversion to B: This model conversion allows the U2B translator tool to convert the model into the B notation where validation and verification tools are available. The translation automatically expresses the constraints of the UML-B model as an invariant property. This defines the multiplicity constraints of each association, and the class membership of instances. The final B machine corresponding to the output model of this stage (Fig.6) is shown in Fig. 7. Note that this domain engineered model really represents a metamodel for any well-defined instance system. The B version allows us to "populate" the metamodel with sample instance data, and check its validity. In Fig. 7 the VARIABLES represent the UML-B classes as container sets for the sample instance data being validated, whilst the INVARIANT carries the metamodel constraint information that the VARIABLES must adhere to.

The first eight invariant conjuncts define the associations as functions between the classes. In some cases the functions are further constrained due to the multiplicities on the associations. Two examples are (i) a total bijection invariant $(>>->)$ generated by $\mathrm{U} 2 \mathrm{~B}$ to reflect the 1 to 1 multiplicity constraints of the association input from CONF to INP, and (ii) the surjectivity $(-->>)$ of association aOut. In some cases extra conjuncts are needed as in the case of dets, which must map to disjoint sets of instances that completely cover the instances of the class DET due to the multiplicity, 1 , at the source end of the association. The last conjunct in Fig. 7 reflects an additional, textual constraint that we added during the domain engineering stage to ensure that all instances of the class ACT are used by one of the association roles that emanate from the class CONF.

Validation by instantiation: To validate the first-cut model we needed to be able to build up the instances it holds in steps ${ }^{5}$. For this task, all classes were given variable cardinality (there is a UML-B clause to define the cardinality and variability of classes) and a constructor was added to each class so that the model could be populated with instances. The constructor was endowed with behaviour (written in $\mu \mathrm{B}$ ) to set any associations belonging to that class to values (i.e. instances of other classes) supplied as parameters.

The developing model was then tested by adding "dummy" instances using the animation facility of ProB and examining the values of the B variables representing the classes and associations in the model to see that they developed as expected. Ini-

\footnotetext{
5 Note that model Fig. 6 is the result of this domain engineering process, and reflects a fixed configuration of DET, CONF etc. elements. Thus the constructors used here are part of this validation process only, and not part of the final system.
} 


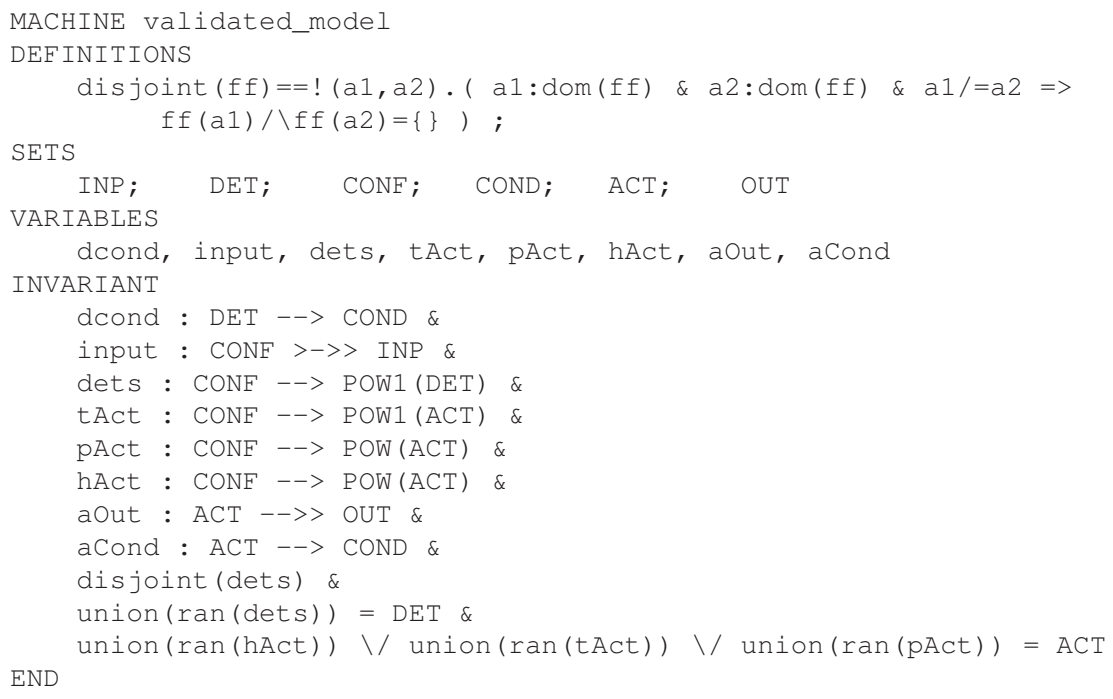

Fig. 7. B Machine

tially, an instance of CONF cannot be added because there are no instances of INP with which to parameterize it. This forces a partial ordering on the population of the model. The INP constructor is available initially because the INP class has no outgoing associations. As soon as an instance of INP is added the multiplicity constraint of the association, input, is violated. ProB provides an indicator to show when the invariant is violated. Observing the invariant violations is an essential part of the validation of the model. Knowing that the model will detect inconsistent instantiations is at least as important as knowing that it accepts consistent ones.

ProB and an example animation: Figure 8 shows ProB being used to validate the final version of the generic model. The top pane shows the $\mathrm{B}$ version of the model generated automatically from the UML-B version (Fig. 6). The centre bottom pane shows the currently available operations (i.e. the constructors we added to the generic model for testing purposes). An operation is only enabled when its preconditions and guards are true. In our model this is when there are unused 'possible' instances left to create and values available to supply as parameters. (Note that, for practical reasons, we limit a class's set of possible instances to a small enumerated set during this stage). An available operation is invoked by selecting it from this pane. Each available operation is repeated in the list for each possible external (parameter) or internal (non-determinism) choice. The left pane shows the current value of each variable as well as an indication of whether the invariant has been violated. The right pane shows the history, i.e. sequence of operations that have already been taken.

We run through the animation shown in Fig. 8:

(1) Initially only the constructors for INP, OUT and COND are available because these classes have no outgoing associations.

(2) An instance o1 of OUT was constructed first as shown at the bottom of the 
history pane. This would have immediately violated the invariant because all such instances must be linked to by the aOut association.

(3) An instance $\operatorname{cf} 1$ of COND was then constructed. This would have enabled the constructors for ACT because an instance of OUT is now available for the new instance to link to via aOut and an instance of COND is available to link to via aCond.

(4) A second instance $\circ 2$ of OUT was constructed.

(5) Finally, an instance a 1 of $\mathrm{ACT}$ was constructed, passing the new instances of OUT and COND for linking via the associations.

(6) The final state of links can be seen in the variables aout and acond representing the associations from ACT. The invariant is (correctly) violated in this state because output $\circ 2$ is not used by an action, disobeying the surjection property of the association.

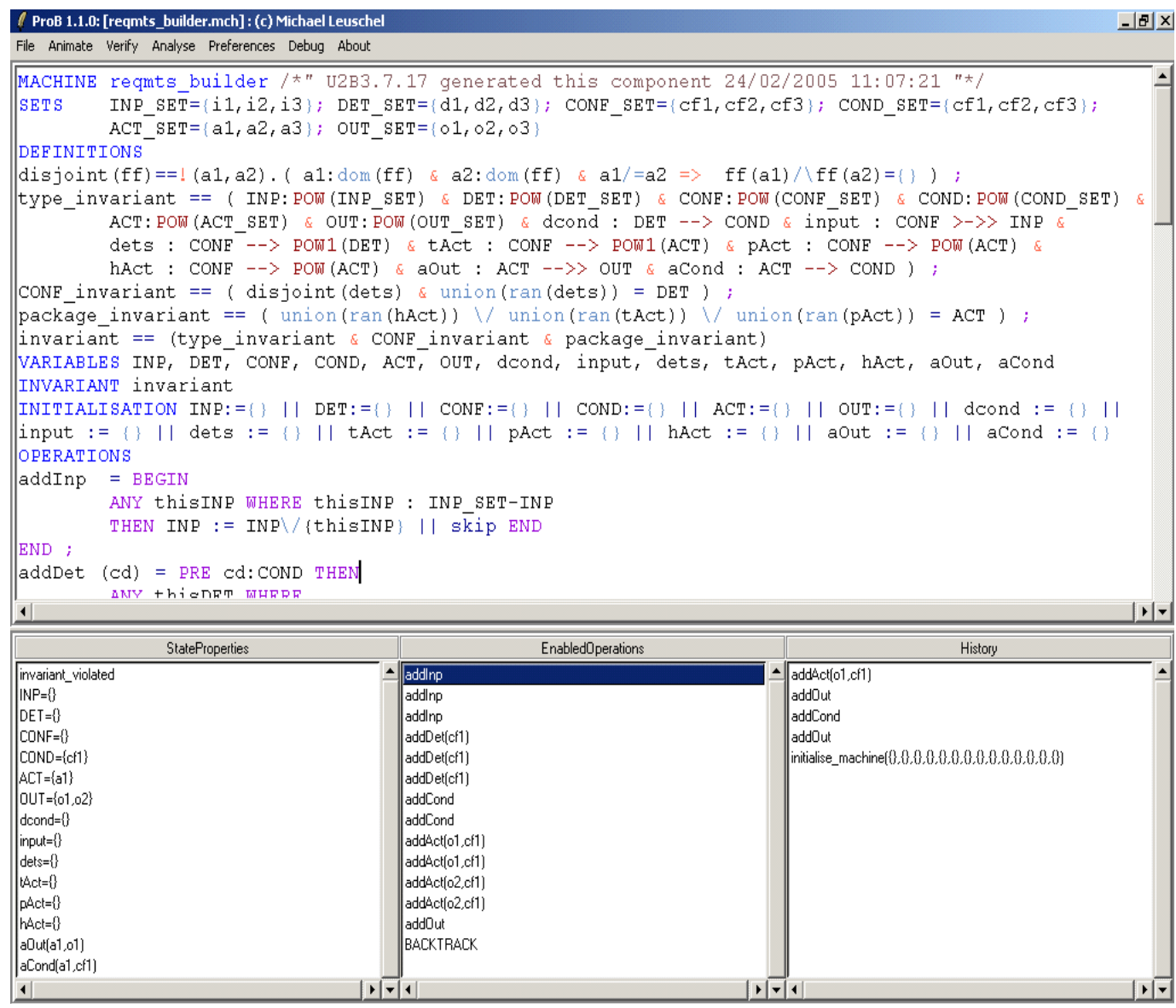

Fig. 8. ProB being used to validate the generic model

The model was rearranged substantially during this phase as the animation revealed problems. Firstly, we discovered some compatibility problems between our example data and the model. The initial model associated each detection with a confirmation (requirement DET_CONF1 in table 3). During animation we discovered that this was a mistake since many related detections may be used on a single input 
all of which should have the same confirmation mechanism. We changed the dets association to $1 \mathrm{CONF}$ to 1-to-many DETs, with the disjointness condition mentioned before. This change had to be reflected in a change to the underlying generic requirement DET_CONF1.

The model was rearranged to associate inputs with confirmations. We also discovered that actions were often conditional, so we added an association from ACT to COND. Apart from these compatibility issues we discovered several changes to the multiplicities of associations. For example, since we had associated sets of actions with confirmations, we did not need a further multiplicity from actions to outputs, thus simplifying the model a little. The most significant change concerned the three associations from $\mathrm{CONF}$ to $\mathrm{ACT}$ where we required each instance of $\mathrm{ACT}$ to be used in at least one of the three associations. Since this is not expressible as a simple multiplicity, a $\mu \mathrm{B}$ invariant was added to the class diagram as an annotation (see Figs. $6,7)$. As for DET_CONF1 above, this validation resulted in further amendments to the narrative generic requirements.

The final model: Thus ProB animation provides a useful feedback tool for validation while domain engineering a reusable model in UML-B. The final version of the generic model is shown in Fig. 6. A confirmation (CONF) is the key item in the model. Every confirmation has an associated input (i nput:INP) to be tested and a number of detections (dets:DET) are performed on that input. Each detection has exactly one enabling condition (dcond:COND). A confirmation may also have a number of actions (hAct:ACT) to be taken while healthy, a number to be taken while confirming ( $t A c t: A C T)$ and a number to be taken once confirmed (pAct:ACT). Each action acts upon exactly one output (aOut:OUT).

Once we were satisfied that the model was suitable, we converted the classes to fixed instances and removed the constructor operations. This simplifies the corresponding B model and the next stage. The associations (representing the relationships between class instances) are the part of the model that will constrain a specific configuration. These are still modelled as variables so that they can be described by an invariant and particular values of them verified by ProB.

\section{Domain engineering - stage template:}

Inputs: Generic requirements specification document (RSD) including first-cut UML model

Approach: Conversion of first-cut UML model to UML-B and then to B. Validation of model by instantiation, using a stepwise constructor approach in ProB animation

Parameters: Dummy instance data in instantiation and animation

Outputs: A validated generic RSD including a validated UML-B generic model

$\mathbf{V} \& \mathbf{V}$ : Using dummy instance data, use ProB to check stage input RSD and unvalidated UML-B model for consistency and required structure 


\section{Application engineering}

Having arrived at a useful generic model for a product range, the model can be put to use by verifying that a particular configuration, or instantiation of the model satisfies its constraints. To illustrate and test this stage we constructed an example instance of a failure management specification for an engine controller, based on existing AT Engine Controls products. This stage represents the verification as per Fig. 3 of a system instance against the classes, associations and invariants of the generic model. The resulting output is a consistent instance model. Note that this method is not limited only to failure management systems; such a generic model and corresponding instances can be developed for any domain where a classassociation style of modelling is applicable and the instantiation is of static configuration data. We return to the question of the generality of the method in the conclusion, section 8 .

Instance specification and incorporation in model: This verification is a similar process to the domain engineering validation, but the focus is on finding and correcting errors in the instance data rather than in the model. The example instance specification was first described in tabular form (see Fig. 10), mimicing the form of table 4 in the RSD. Each class is represented by a separate table with properties for each entry in the table representing the associations owned by that class. To verify its consistency, the tabular form was translated into class instance enumerations and association initialization clauses attached to the UML-B class model. This one-shot initialization process in UML-B is in contrast to the stepwise instance-constructor process of the previous stage. Initially, table translation to UML-B was done manually, which was tedious and error prone.

Verification with ProB: ProB was then used to check which conjuncts of the invariant were violated by the example instantiation. The invariant is a predicate that expresses all the constraints of the generic model: instances belong to classes, association links satisfy the multiplicity constraints of their associations and any further annotated invariant predicates that may involve several associations. To check the invariant is satisfied by the instance mode, all that is needed is to invoke the initialization in ProB. Fig. 9 shows the "analyse invariant" facility of ProB being used to identify an error (each line represents a conjunct in the invariant from Fig. 7). Several conjuncts are violated ( $=\mathrm{false}$ ); all are constraints on associations involving the class, ACT. For example, the second of these (aOut) states that every ACT must be associated with exactly one OUT, and that every OUT must be associated with one or more ACTs ${ }^{6}$. Examining the initializations of these associations reveals that links had not been added for action act 1310 . Several iterations were necessary to eliminate errors in the tables before the invariant was satisfied (all conjuncts =

$\overline{6}$ This is what is meant by "-- >>" in B in Fig. 7, and by "TotalSurjection" in ProB in Fig. 9. 
true).

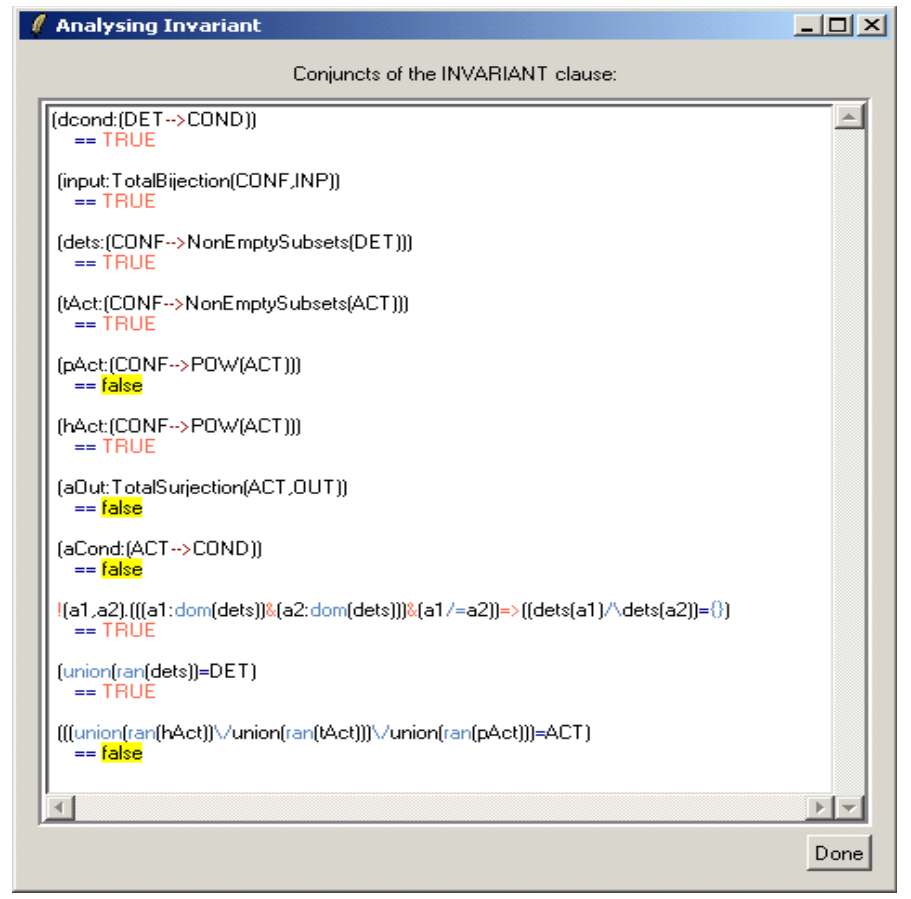

Fig. 9. ProB being used to verify the example application

We found that the analyse invariant facility provided some indication of where the invariant was violated (i.e. which conjunct) but, in a data intensive model such as ours, it is still not easy to see which part of the data is at fault. In the example above, any ACT without initialized associations would cause the aOut conjunct to go false. In general there are many data instances of a given class. What is required from such tool-supported verification is the automated production of a data counterexample to the conjunct.

\subsection{Requirements Manager(RM): A tool for instance data management}

To address the problems found with using ProB to verify instantiation data, we developed a tool that interfaces with the UML drawing tool to automate management and verification of instance configuration data. The tool was developed as an Eclipse plug-in by a student group ${ }^{7}$. The tool provides an extension to the Rational Software Architect UML modelling tool (which is also based on Eclipse). Menu extensions are provided to operate the tool from the class diagram of the generic model, so that a database repository can be generated based on the classes and their associations. Instance specification data, in the form of class instances and association links, can then be 'bulk uploaded' directly from Excel configuration files such as Fig. 10. This avoids the tedious and error prone process of manually populating

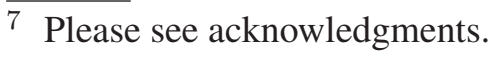


the class diagram. The figure shows a sample of the configuration data that was loaded automatically by the tool.

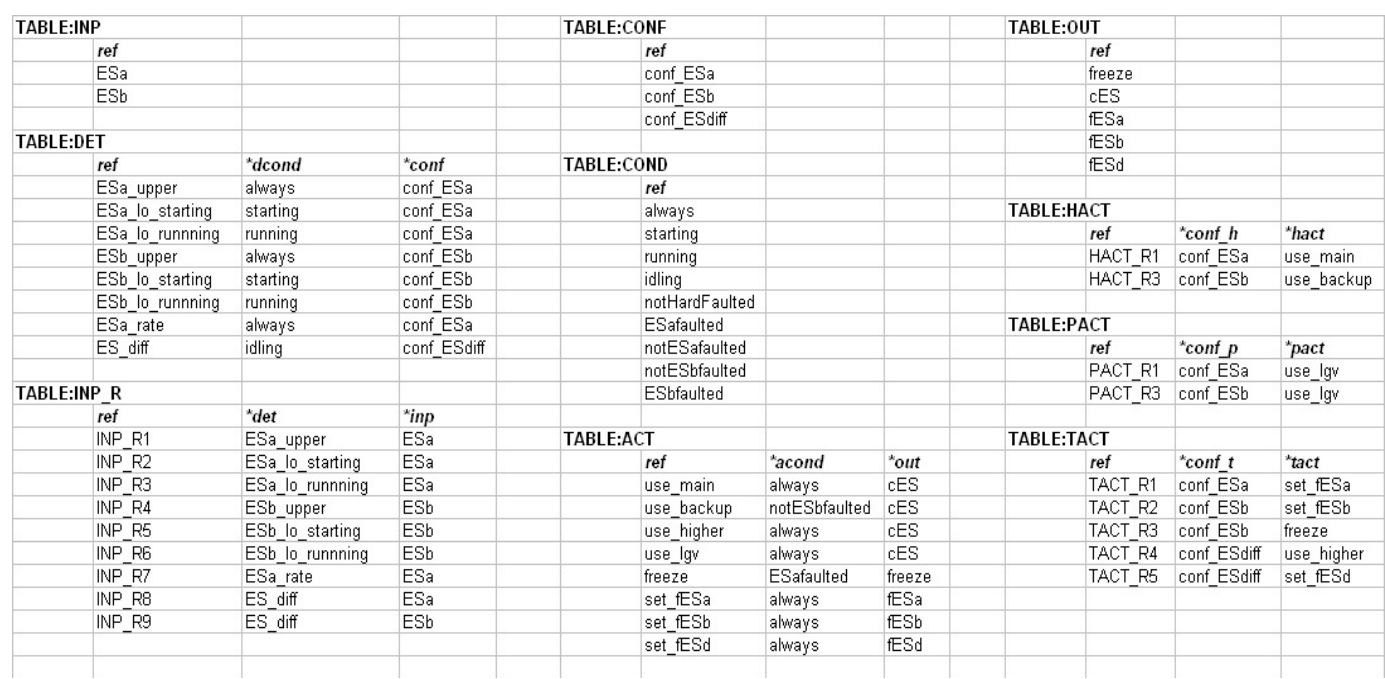

Fig. 10. Sample instance configuration data

Some types of verification errors (such as mismatches between the class diagram and tables and referential integrity errors) may prevent the data from being uploaded. The tool reports these errors giving the exact details of the counter example to the constraint represented by the model. Fig. 11 shows the error view provided by the tool. Several such failures are shown in the lower half of the error view. For example, the first of these errors states that an instance, ESa, from the data cannot be inserted into class, INP, because an instance of that name already exists.

If the configuration data has none of these errors it is loaded into the database schema and further verification is performed by checking class diagram constraints, such as multiplicity constraints on associations. These multiplicity errors are shown in the upper half of the error view in Fig. 11. The error messages identify the particular data instances that violate the multiplicity constraint giving sufficient information to pinpoint the problem. That is, a counter example is given, solving the problem we experienced with the ProB model checker. The error can then be corrected either by editing and re-loading the configuration data, or by editing the database from the class diagram. For the latter, an extension to the class pop-up menu is provided, giving direct access to the relevant database table as shown in Fig. 11 where a multiplicity error is being corrected. Although the tool identifies the nature of the error more precisely than ProB (by giving all counter examples whereas ProB only identified which constraint was violated), it may still be difficult to find the correct solution. For example, in Fig. 11, the error message gives the counter example that there is no association link between the instance ES_diff:DET and an instance of CONF. This contravenes the multiplicity constraint (1) at the target end of the association, conf. Knowledge of association links throughout the class diagram is needed to find a correction. In future work we intend to provide tools to visualise the transitive association links for a given set of class instances by 


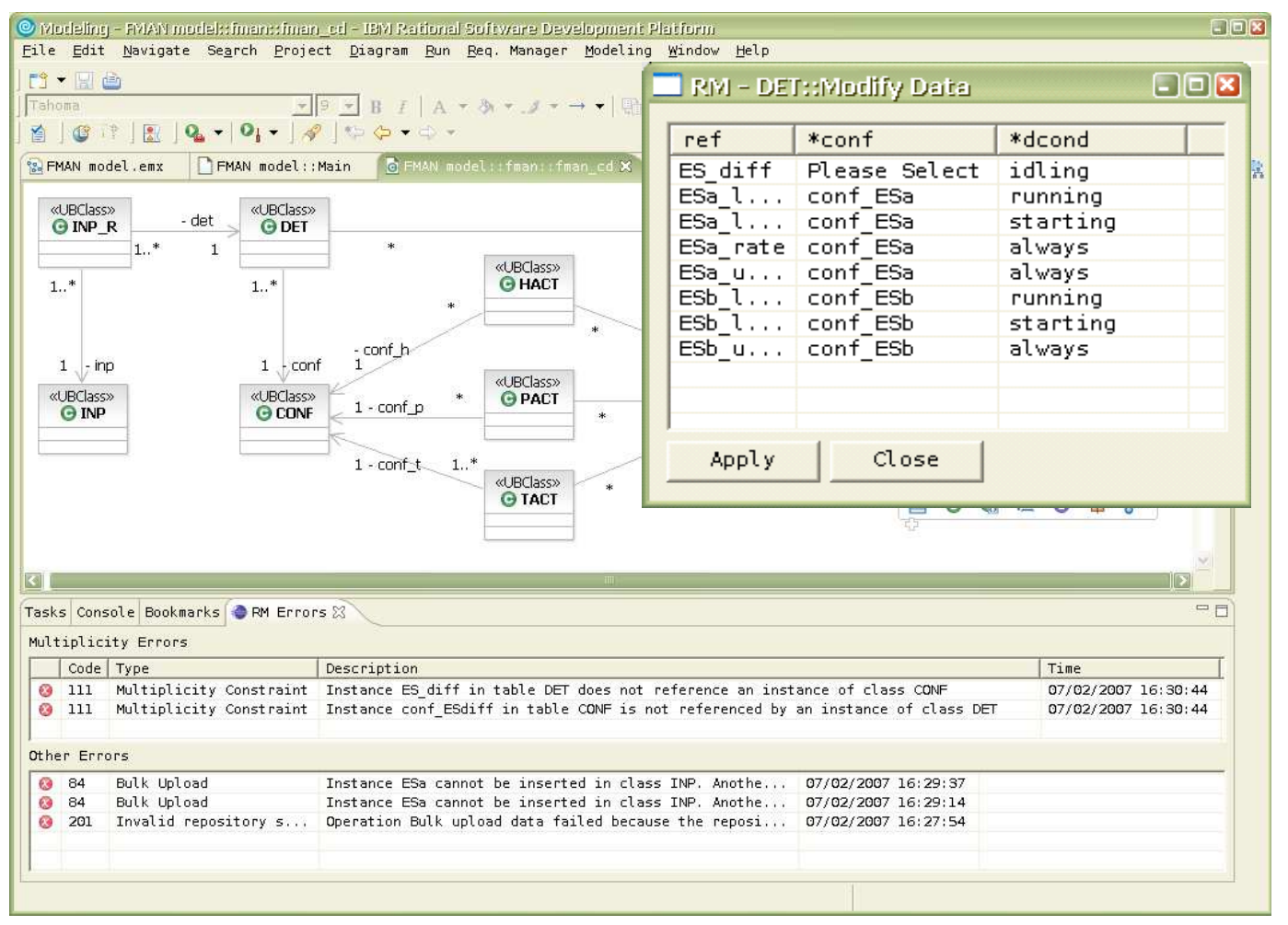

Fig. 11. Tool screenshot with error view and update window

automatically generating object diagrams from the database.

The ability to modify data via the class diagram enables individual class instances and association links to be added to an existing (or developing) configuration. The requirements engineer can invoke the Requirements Manager(RM) tool at selected points (when the data is expected to be in a consistent state) to check the configuration satisfies the generic constraints of such systems. A limitation of the database approach to managing configuration data is that many to many association relations can not be represented in database schema. In order to represent many to many associations we had to add intermediate linking classes (see INP_R inserted between INP and DET and HACT, PACT and TACT between CONF and ACT). In future versions of the tool we intend to hide this representation mismatch from the user.

The RM tool has been developed as an Eclipse plug-in to integrate with the RODIN project toolset including the UML-B drawing tool, U2B translator, ProB, B prover and $B$ database. In parallel with the development of RM, the U2B tool has been redeveloped in Eclipse to accept input models based on the UML2 metamodel (upon which RSA models are based). A UML2 profile has been developed to extend the UML notation and provide relevant property fields to accept information such as the configuration data. Once the configuration data has been successfully verified RM can be used to populate the UML-B stereotype properties. This utilises the instances property attached to classes and the value property attached to associations. These values are utilised by U2B when it produces a B version of the model. Fig. 
12 shows the stereotype property value for an association after population by RM.

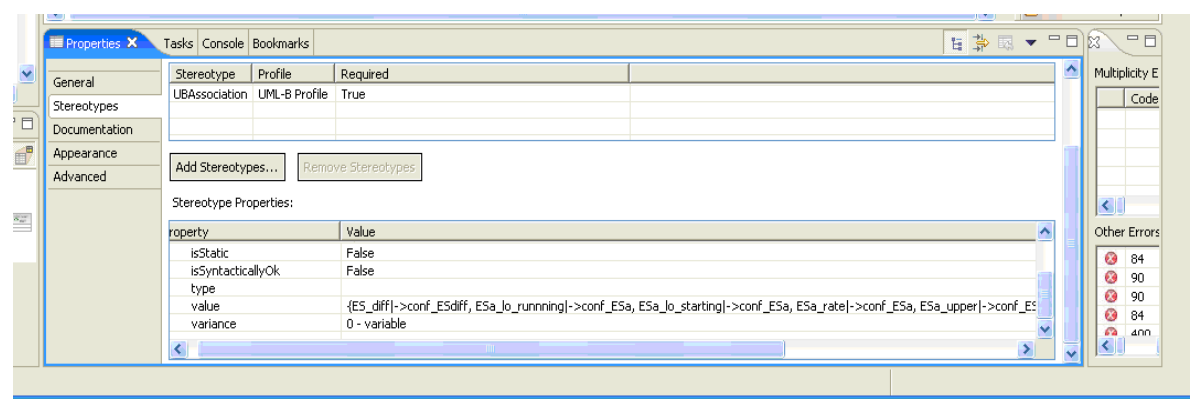

Fig. 12. Tool screenshot with properties view

Fig. $13^{8}$ illustrates the process of using the RM tool ${ }^{9}$. Once a class diagram has been loaded (loadCD), the database schema can be generated (generateDBS). If this fails, for example if the class diagram contains elements that are not supported, a new class diagram must be loaded (or the current one modified). If it succeeds, the state DBSGenerated has an invariant dbs_is_correct representing that a valid schema is available for the class diagram. From this state data can be bulk loaded, loadDT, which may fail due to referential integrity problems or succeed and enter the state DTLoaded. The invariant, dt_preverifies, for this state, represents that data has no such errors. The data can now be checked (checkDT) for other errors, such as multiplicity constraint violations. If there are no such errors (dt_verifies) the data can be used to populate the class diagram (generateoBS). At any time, if the class diagram is modified (loadCD), the schema must be regenerated and new data loaded.

\section{Application engineering - stage template:}

Inputs: Validated generic requirements specification document (RSD) including validated UML-B model

Approach: Verification of instance data by one-shot initialization in ProB. Requirements Manager tool for system instance specification repository and verification, with data counterexamples

Parameters: Sample instance data from sample instance system tables in RSD

Outputs: A verified system instance model

$\mathbf{V} \& \mathbf{V}$ : Verification on instance data against validated generic model using ProB

\footnotetext{
8 The symbol $\neg$ means logical negation.

9 This statechart corresponds to a partial B model of the RM tool, which has been fully model-checked in ProB.
} 


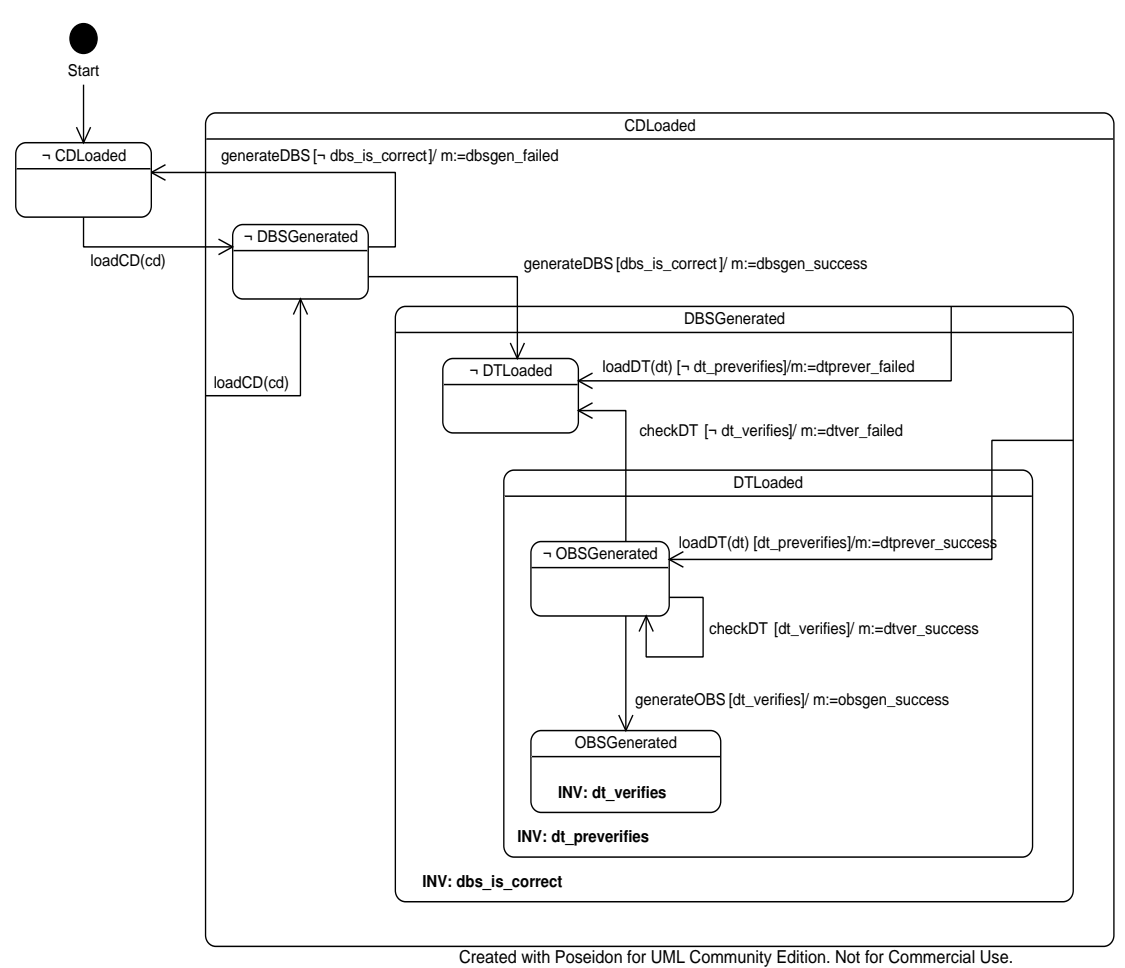

Fig. 13. Statechart for RM tool

\section{Industrial User View}

The techniques described in this paper include several areas which will be of interest to industry.

- The identification and formal validation of a generic requirement model for a given problem domain.

- A technique to formalize UML and benefit from formal validation and verification tools and methodologies

- A technique to automatically verify the instantiation of a generic model with realistic large scale data appropriate for product line engineering.

The class of problems targeted by our method have a relatively simple architectural structure of common functions but a complex pre-determined instantiation of these functions. With such problems, the identification of the underlying common functional architecture is crucial to identify generic requirements and make the problem manageable. Our industrial partner, ATEC, had no prior experience with formal specification techniques but with support and guidance was able to perform the modelling and use the associated tools. The next stage, adding behavioural details, would entail textual specification of operation guards and actions requiring more extensive understanding of $\mu \mathrm{B}$. Work is underway in the RODIN project to improve the accessibility of UML-B by increasing integration with the 
underlying B toolset. The animation facility of the ProB tool is particularly useful to demonstrate behaviour to the domain user and, as used here, did not require knowledge of B to understand it. However it is envisaged that to demonstrate the behavoural development of the model the animation may need to be supported by model documentation and an industrial strength GUI to provide a domain expert a clearer understanding of the model when executing scenarios. With support from the instantiation database tools the technique contributes towards product line development through its ease of instantiating realistic scale of requirements data.

\section{Conclusion and related work}

\subsection{Related work in context}

Parnas' prescient early work [35] characterized three types of approach to the development of software product lines, or "program families" - (i) syntactic modification, (ii) modular specification, and (iii) refinement. At that time, type (i) involved the development of a complete program, followed by the production of variants by modifying the original program. Since then this type of approach has been elaborated through process phases e.g. requirements, architecture, and through the structuring of artefacts of those phases. Type (ii) has grown into today's componentbased software engineering (CBSE) approaches such as [16,18,43]. All types of approach involve a domain engineering activity that captures the requirements that all family instances must share - the commonalities - and the requirements that vary between instances - the variabilities [14] - into a generic, reusable software resource. This is followed by an application engineering activity that uses this resource to generate the specific instance systems as necessary. Most product line work assumes an early domain analysis activity, e.g. [37], for gathering and structuring all relevant information from the application domain to support the development of such a generic, reusable software resource.

It is noteworthy that the Parnas' approach type (i) remains dominant today, e.g. $[36,19,26,25]$. In this type of approach, application engineering deploys an instance derivation process against generic models/architectures and specific components/interfaces and variation points, to generate an instance system: an elaborate process of syntactic modification. In [36] an "Orthogonal Variability Model" models variabilities, their constraints and variation points as first-class citizens, separately from the generic architecture of commonalities. The maturity of the component model in the picture may merge Parnas' approach type (i) into type (ii).

Some logic-based and formal methods techniques have been proposed for software product lines. Validation of logically-defined requirements and constraints on the variabilities (e.g. $\operatorname{var}_{1}(i) \Rightarrow \operatorname{var}_{2}(i) \wedge \neg \operatorname{var}_{3}(i)$ for instance $i$ ) can be per- 
formed [30]. Feature models defined with differing degrees of genericity, binding into the software construction process at different points, can be validated similarly [46]. Formal models also allow formal verification to happen, e.g. feature modelchecking [46] and product line architectural model-checking for commonalities of robustness and fault-tolerance [28].

In particular, formal refinement-based approaches (Parnas' third type of approach above) largely remain to be applied to software product lines. The notion of refinement by trace restriction [45] is defined by the structured deletion of steps from traces of the "maximal" instance, which exhibits all possible behaviours of all instances. This proposal is applicable to a product line where all components are defined at the same level of abstraction, and there are no variability constraints. The generative feature-oriented programming approach of [9] is built on a notion of refinement whose meaning is closer to object-oriented extension, or inheritance, rather than the classical refinement of Hoare, He, Back et al $[24,7,8]$.

Our approach is of Parnas' type (i) - the class diagram of Fig. 6 is the generic product line model. On input of data for a system instance, the RM database checks that the data satisfies the dependencies of the class diagram, and facilitates user "debugging" of erroneous data. The SQL database DECIMAL tool of [34] performs a similar task, although not in a formal verification context. The system instance is specified by "populating" the UML-B stereotype with this verified instance data, and $\mathrm{U} 2 \mathrm{~B}$ then combines the generic and instance information into an instance B specification, for further formal verification with ProB and theorem provers. What distinguishes our methodological and tooling work for product lines is its integration with a leading language and method for formal specification, refinement and verification, Event-B.

Future work using UML-B inheritance and refinement to elaborate system behaviour will relate the approach to Parnas' type (iii) - refinement. For example, the abstract model now specifies generic detection behaviour in terms of checking an input-derived value against a limit. A refinement specializes this behaviour for magnitude, rate, multiple etc. detection types. Thus we anticipate that the instance data population (application engineering) stage will become more elaborate, populating a graph of refinements, rather than a single model as at present. The Event-B method under development by project RODIN will provide mechanisms for composition and decomposition with refinement, to support scalable development. The generic instantiation mechanism of Event-B, whereby a model can be made generic with respect to one or more configurable contexts, will afford a component-like form of reuse.

The case for formal and refinement approaches to SPLE is reinforced by an industrial experience analysis [15] of EU-IST project ConIPF [38], where product-line infrastructure failed to deliver the time and effort savings originally hoped for. In this iterative component-based approach including significant human interaction, it 
was found that false positives in component selection, human errors in resolving variabilities, unforseen later consequences of early variant selection, problems in resolving provided/required component interfaces were all excessive. This boiled down to the issues of inadequate understanding, modelling and analysis of product line complexity and implicit properties, i.e. under- or undocumented variability constraints. Component interfaces were only specified syntactically (parameter types), and not semantically (e.g. pre- and postconditions). Semantic specification of interfaces and the performance of formal anaysis that would be thus enabled, would reduce these problems. This experience suggests that more thorough formal modelling and analysis, with the layered elaboration of complexities and constraints through a methodical refinement process, would be beneficial.

\subsection{Evaluation}

We found that the domain analysis stage was useful in approximating abstractions for generic requirements. Most of the requirements taxonomy from this stage survived subsequent analysis through to the final generic requirements model showing that the conceptual abstractions were valid and useful. However, the detail in the model was altered several times during the subsequent stages. This indicates that domain analysis alone is insufficient for precise specification and that some form of validation is essential.

During the domain engineering stage, many changes consisted of adjusting the multiplicities on association relationships. Although it was the association that was modified, the conceptual change was to the classes. That is, the semantics of the classes (representing the abstract taxonomy of requirements) were found to be less than ideal. When our perception of the semantics changed (which, of course, doesn't require a change to the diagram) the associations, or their multiplicities, were no longer valid. For example we changed the ACT class from representing a set of assignments on output variables to represent a single assignment to one output. The lesson learned is that many of the changes during this stage result from a better understanding of the underlying semantics of the domain requirements and the most efficient way to represent them, rather than directly from consistency checking. This understanding was achieved by progressive introduction of instances in order to exercise consistency checking. We were gradually adding more data to the model to deliberately break its constraints. An interesting lesson is that one can sometimes learn more from exploring how data breaks constraints than from data that satisfies them. Another lesson, obvious but worth stating, is that the model should be fully covered during validation: every association, and every kind (one-one, one-many, total ) of link in an association, should be instantiated at least once.

During the application engineering stage we found that, for the kinds of systems 
that we are interested in, it is not sufficient to know that the model is incorrect. In large configurations it is equally important to have some indication of where the data is incorrect. In general, it is not possible for a model checker to determine this because the inconsistent data cannot be decisively analysed; the inconsistency could lie in any part of the data. For our scenario, where we populate sets and relations between sets, we were able to provide a tool that identifies each of the elements that contravened the constraints. However, although this gives clues, in some situations, especially when there are many such contraventions, it could still be difficult to find a solution. In ongoing work we are looking at ways to visualize the data to assist in discovering solutions. In building this tool we have found that the "impedance mismatch" between object-oriented and relational database representations, is problematic. To cater for many to many relationships we were forced to introduce intermediate classes. We would therefore recommend instead using an object-oriented repository to store entity-relationship model data.

\subsection{Future work}

As indicated before, the method and tools presented are work in progress. The domain analysis stage revealed key requirements issues, which were better understood by then considering their rationale. At the moment, however, these are not enforced by the generic model. Key issues are higher level requirements that could be expressed at a more abstract level from which the (already validated) generic model is a refinement. The generic model could then be verified to satisfy the key issue properties by proof or model checking. This matter is considered in [41] which gives an example of refinement of UML-B models in the failure management domain. The domain analysis process of Fig. 2 would then be elaborated as shown in Fig. 14.

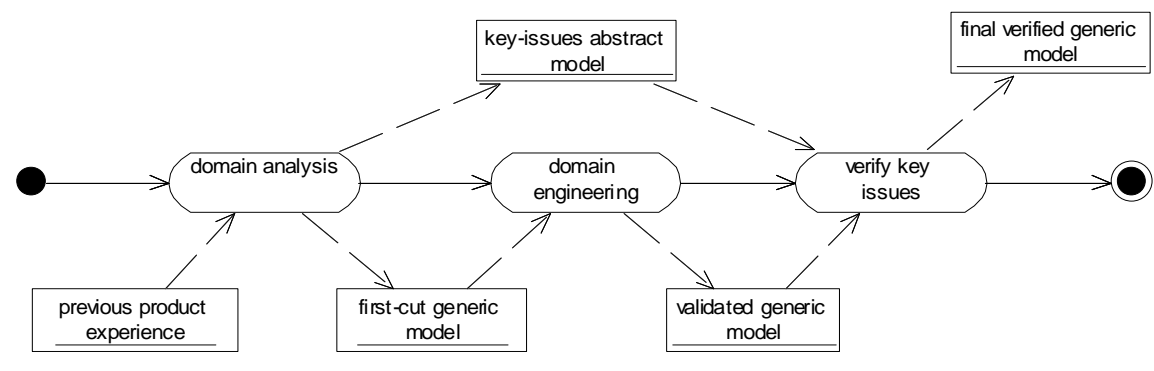

Fig. 14. Elaboration of domain analysis process to show refinement of key issues

Behavioural specification via refinement: The specification and elaboration of behaviour in UML-B is a stepwise process using the B form of classical refinement [2]; this is ongoing work for the FMS case study. It transpires that the key issues of table 5 can be treated as specification-level features, that help structure the behavioural specification activity. For example, the detection feature (key issue (1)) has behaviour associated with classes DET and INP in the validated UML-B model 
of Fig. 6. The first, most abstract behavioural model for these classes involves the events of reading to read an INP, eval to evaluate the detection predicate for DET over associated INPs, and pass and fail for DET. This abstract description is highly nondeterministic and is concerned only with valid event sequencing. For detection, the main sequencing constraints are that (i) reading is always enabled, (ii) eval is only enabled when fresh readings are available for all INPs for the current DET, and (iii) pass and fail are only enabled once eval has produced an appropriate value.

Subsequent refinement models then elaborate these behaviours, reducing the nondeterminism into algorithmic structure, and adding supporting data infrastructure. Following the detection example above, for a range test, the next refinement would elaborate the pass/fail judgment mechanism by introducing a guard to determine whether the INP expression is in or out of range.

The confirmation feature (key issue (2) of table 5) has behaviour associated with classes DET and CONF in Fig. 6. The abstract confirmation event writeHist for CONF is enabled once all associated DET tests have run; an appropriate action healthy, confirming, or confirmed is then taken, depending on a judgment made on the confirmation history. That judgment is not specified in the abstract description but is elaborated in refinement in terms of the fault counts of table 4.

Behavioural validation of instance models: Further development is required to validate instance models, as per section 3, Fig. 3. Whilst an instance model can be verified against the constraints specified in the generic model (i.e. that it is a valid instantiation of the generic model), it may be the wrong instance, i.e. it may specify the wrong run-time behaviour. The development of a further method stage to validate behaviour for a specific instance configuration is now under way. This stage will involve ProB animation of behaviour against the emergent refinement models.

Applicability of method: What we have proposed in this paper is a method targetted at product lines, for the production and verification of (i) the generic requirements specification and (ii) the system instance specification. The method is focussed on the construction of specifications expressible as UML-B class diagrams. The class diagram is a simple but highly expressive modelling tool, with a long pedigree going back to Chen's Entity-relationship modelling [13]. We use the class diagram as a metamodel for valid static instance configurations and believe that the failure management application is a convincing demonstration of its expressiveness. FM is but an example of potential application domains. We assert that the method will be applicable to any product-line domain with significant static data configuration requirements. We briefly consider two examples.

An industrial process control system comprises a variety of hardware components, 
interacting in a complex manner with each other and with software. The static configuration for requirements engineering with the method is precisely many instances of many classes of hardware component, each with operating characteristics, and each in operating relationships with others. For example, the process controller needs to know the connectivity of a fluid processing line, say from a containment vessel, through exit valve and flow pipe to a reaction vessel, in order to monitor and control fluid flow. It also needs to know the volume and pressure capacity of pipe and vessels. For control of the containment vessel it needs to know the relief valve, pressure and low-level sensor fit. The method is applicable.

A point-of-sale terminal network comprises many terminals, network connections of various types (e.g. ethernet, wireless LAN, WAN), servers and other processing units. Servers might be connected pairwise on ethernet for redundancy, these pairs being physically distributed and WAN-connected. There will be terminal - server pairings for particular functions. Each terminal might be assigned a backup terminal with a requirement that the server connectivity of the terminals is the same: this is an example of a complex constraint not expressible as a simple association, but expressible as a $\mu \mathrm{B}$ invariant in the UML-B model. Again, this example represents a static configuration where the method is applicable.

Improved tool support: Section 6 identified the need for finer-grained diagnosis of invariant violation in ProB. ProB could be enhanced to provide, for example, a data counterexample causing an invariant violation; this is precisely what the RM tool does. However, a related need is for verification and debugging support for bulk upload (as well as incremental development) of instance data. For example, when told that a CONF is missing an associated DET, the engineer will need to drive a selective visualization of the instance data - perhaps in the first instance, all existing DET - CONF associations - in order to "debug" and correct that data.

The current U2B tool leads to a separation between the modelling language (UMLB) and the verification and validation language (B). In future work, we will provide better integration and feedback of verification results to the source models based on a new, extensible, version of the B tools. However, even without this integration, UML-B provides benefits in the form of model visualisation, and efficient model creation and editing compared to textual $\mathrm{B}$.

A partial formal specification in B of the RM tool has been written and modelchecked in ProB. This work is ongoing to add assurance about tool correctness.

\section{Acknowledgements}

We are grateful to ECS students Ledina Hido, Robert Stops and Martin Ross for their work developing the Requirements Manager tool, and to Ledina for her work 
on the tool specification and verification in B.

\section{References}

[1] EUROCAE ED12B - Software considerations in Airborne Systems and Equipment Certification. http://www.eurocae.org.

[2] J.-R. Abrial. The B-Book: Assigning Programs to Meanings. Cambridge University Press, 1996.

[3] J.-R. Abrial. Formal Method course, Part I: Introduction. 2005.

[4] J.-R. Abrial and ClearSy. http://www.atelierb.societe.com/index_uk.htm, 1998. Atelier-B.

[5] P. Amey. Dear sir, Yours faithfully: an everyday story of formality. In F. Redmill and T. Anderson, editors, Proc. 12th Safety-Critical Systems Symposium, pages 3-18, Birmingham, 2004. Springer.

[6] B. Arief et al. Traceable requirements document for case studies. Technical Report Deliverable D4, EU Project IST-511599 - RODIN, February 2005. http://rodin.cs.ncl.ac.uk/.

[7] R.J.R. Back. On correct refinement of programs. Journal of Computer and System Sciences, 23:49-68, 1981.

[8] R.J.R. Back. A calculus of refinements for program derivations. Acta Informatica, 25:593-624, 1988.

[9] D. Batory. A science of software design. In AMAST 2004, volume 3116 of LNCS, pages 3-18. Springer, 2004.

[10] C.M. Belcastro. Application of failure detection, identification, and accomodation methods for improved aircraft safety. In Proc. American Control Conference, volume 4, pages 2623-2624. IEEE, June 2001.

[11] G. Booch, I. Jacobson, and J. Rumbaugh. The Unified Modeling Language - a Reference Manual. Addison-Wesley, 1998.

[12] D. Cansell, J.-R. Abrial, et al. B4free. A set of tools for B development, from http://www.b4free.com, 2004.

[13] Peter Pin-Shan Chen. The entity-relationship modeltoward a unified view of data. 1(1), March 1976.

[14] J. Coplien, D. Hoffman, and D. Weiss. Commonality and variability in software engineering. IEEE Software, pages 37-45, November/December 1998.

[15] S. Deelstra, M. Sinnema, and J. Bosch. Experiences in software product families: Problems and issues during product derivation. In SPLC 2004, volume 3154 of LNCS, pages 165-182. Springer, 2004. 
[16] S. Deelstra, M. Sinnema, and J. Bosch. Product derivation in software product families: a case study. Journal of Systems and Software, 74:173-194, 2005.

[17] B. Dehbonei and F. Mejia. Formal development of safety-critical software systems in railway signalling. In M.G. Hinchey and J.P. Bowen, editors, Applications of Formal Methods, chapter 10, pages 227-252. Prentice-Hall, 1995.

[18] D. D'Souza and A. Wills. Objects, Components and Frameworks with UML. AddisonWesley, 1998.

[19] S.R. Faulk. Product-line requirements specification (PRS): an approach and case study. In Proc. Fifth IEEE International Symposium on Requirements Engineering. IEEE Comput. Soc, Aug. 2001.

[20] M. Fayad and D. Schmidt. Object-oriented application frameworks. Communications of the ACM, 40(10):32-38, Oct. 1997.

[21] Radio Technical Commission for Aeronautics. RTCA DO $178 B$-Software considerations in Airborne Systems and Equipment Certification. http://www.rtca.org.

[22] M. Gogolla, J. Bohling, and M. Richters. Validation of UML and OCL models by automatic snapshot generation. In G. Booch, P. Stevens, and J. Whittle, editors, Proc. 6th Int. Conf. Unified Modeling Language (UML'2003), volume 2863 of LNCS. Springer, 2003.

[23] M. Gogolla and M. Richters. A UML-based specification environment, 2006. http://www.db.informatik.uni-bremen.de/projects/USE/.

[24] Jifeng He, C.A.R. Hoare, and J.W. Sanders. Data refinement refined. In B. Robinet and R. Wilhelm, editors, ESOP86: European Symposium on Programming, volume 213 of $L N C S$. Springer, 1986.

[25] W. Lam. Achieving requirements reuse: a domain-specific approach from avionics. Journal of Systems and Software, 38(3):197-209, Sept. 1997.

[26] W. Lam. Creating reusable architectures: Initial experience report. ACM SIGSOFT Software Engineering Notes, 22(4):39-43, July 1997.

[27] M. Leuschel and M. Butler. ProB: a model checker for B. In K. Araki, S. Gnesi, and D. Mandrioli, editors, Proc. FME2003: Formal Methods, volume 2805 of LNCS, pages 855-874, Pisa, Italy, September 2003. Springer.

[28] R. Lutz and G. Gannod. Analysis of a software product line architecture: an experience report. Journal of Systems and Software, 66:253-267, 2003.

[29] R. Macala, L. Jr. Stuckey, and D. Gross. Managing domain-specific, product-line development. IEEE Software, pages 57-67, May 1996.

[30] M. Mannion. Using first-order logic for product line model validation. In SPLC2, volume 2379 of $L N C S$, pages 176-187. Springer, 2002.

[31] C. J.-R. Abrial, and L. Voisin. Event-B Language. Technical Report Deliverable 3.2, EU Project IST-511599 - RODIN, May 2005. http://rodin.cs.ncl.ac.uk. 
[32] B. Meyer. Object-Oriented Software Construction. Prentice Hall, 1997.

[33] UK Ministry of Defence. Def Stan 00-55: Requirements for safety related software in defence equipment, issue 2. http://www.dstan.mod.uk/data/00/055/02000200.pdf, 1997.

[34] P. Padmanabhan and R. Lutz. Tool-supported verification of product line requirements. Automated Software Engineering, 12(4):447-465, October 2005.

[35] D. L. Parnas. On the design and development of program families. IEEE Transactions on Sofkvare Engineering, SE-2(1):1-9, March 1976.

[36] K. Pohl, G. Boeckle, and F. van der Linden. Software Product Line Engineering Foundations, Principles, and Techniques. Springer, Heidelberg, 2005.

[37] R. Prieto-Diaz. Domain analysis: An introduction. ACM SIGSOFT Software Engineering Notes, 15(2):47-54, 1990.

[38] ConIPF project. Configuration of industrial product families. http://segroup.cs.rug.nl/conipf.

[39] S. Schneider. The B-Method. Palgrave Press, 2001.

[40] C. Snook and M. Butler. U2B - a tool for translating UML-B models into B. In J. Mermet, editor, UML-B Specification for Proven Embedded Systems Design, chapter 5. Springer, 2004.

[41] C. Snook, M. Butler, A. Edmunds, and I. Johnson. Rigorous development of reusable, domain-specific components, for complex applications. In J. Jurgens and R. France, editors, Proc. 3rd Intl. Workshop on Critical Systems Development with UML, pages 115-129, Lisbon, 2004.

[42] C. Snook, I. Oliver, and M. Butler. The UML-B profile for formal systems modelling in UML. In J. Mermet, editor, UML-B Specification for Proven Embedded Systems, chapter 5. Springer, 2004.

[43] C. Szyperski, D. Gruntz, and S. Murer. Component Software - Beyond Object-Oriented Programming. Addison-Wesley / ACM Press, 2002.

[44] W. Tracz. DSSA (Domain-Specific Software Architecture) pedagogical example. ACM Software Engineering Notes, pages 49-62, July 1995.

[45] A. Wasowski. Automatic generation of program families by model restrictions. In SPLC 2004, volume 3154 of LNCS, pages 73-89. Springer, 2004.

[46] W. Zhang, H. Zhao, and H. Mei. A propositional logic-based method for verification of feature models. In ICFEM2004, volume 3308 of LNCS, pages 115-130. Springer, 2004. 